\title{
Notas sobre el tratamiento clínico de la psicología cognitivo-comportamental: un análisis de la depresión
}

\author{
Notes on the clinical treatment of \\ cognitive-behavioral psychology: an \\ analysis of depression
}

\author{
Clayton Rolim Teixeira' 1 (1) \\ Thamires Pereira Alves ${ }^{2}$ (1) \\ Maria Waldilene Sousa Teixeira ${ }^{3}$ (1)
}

Tadeu Lucas de Lavor Filho 4

Lielton Maia Silva ${ }^{5}$ Antoniel dos Santos Gomes Filho 6 (1)

${ }^{1-3,5}$ Centro Universitário Vale do Salgado (Icó). Ceará, Brasil. claytonrolim@hotmail.com, thamirespereiraalves@hotmail.com, waldilenesouza@fvs.edu.br, lieltonmaia@fvs.edu.br ${ }^{4}$ Autor correspondente. Centro Universitário Vale do Salgado (Icó). Universidade Federal do Ceará (Fortaleza). Ceará, Brasil. tadeulucaslf@gmail.com ${ }^{6}$ Centro Universitário Vale do Salgado (Icó), Universidade Federal de Campina Grande (Cajazeiras). Ceará/Paraíba, Brasil. antoniel.historiacomparada@gmail.com
RESUMEN | INTRODUCCIÓN: El tratamiento psicoterapéutico desde la perspectiva de la Psicología a través de la Terapia Cognitivo-Conductual (TCC) tiene en su cuna clínica una mirada refinada sobre los fenómenos de la cognición. OBJETIVO: Esta investigación analizó la perspectiva clínica de la terapia cognitivoconductual (TCC) que aparece en la literatura científica publicada sobre el tratamiento de la depresión. MÉTODO: A través de una revisión bibliográfica sobre el tema en la Plataforma Académica de Google y la Biblioteca Electrónica Científica (SciELO) en los años 2010-2020. RESULTADOS Y DISCUSIONES: La literatura muestra que la resolución de los atolladeros de una manera más asertiva, la reestructuración cognitiva y la directiva y el proceso psicoterapéutico enfocado se entienden como una contribución a la eficacia del tratamiento. Se observó que, entre las técnicas existentes en la TCC, algunas son más eficaces, a saber: conceptualización cognitiva, registro de pensamientos disfuncionales, psicoeducación, reestructuración cognitiva, cuestionamiento socrático, capacitación en aptitudes sociales y deberes. CONSIDERACIONES FINALES: En el presente estudio se abordó la prevalencia de los trastornos depresivos, sus perspectivas de desarrollo, signos y síntomas, así como la gestión del tratamiento, y puede inferirse que la TCC y los instrumentos técnicos que utiliza corroboran el progreso de la psicoterapia y la reestructuración cognitiva de los individuos depresivos, atenuando los síntomas, en asociación con el uso de drogas psicotrópicas.

PALABRAS CLAVE: Psicología cognitivo-comportamental. Depresión. Tratamiento clínico.

\begin{abstract}
INTRODUCTION: The psychotherapeutic treatment from the perspective of Psychology through Cognitive-Behavioral Therapy (CBT) has in its clinical cradle a refined look at the phenomena of cognition. OBJECTIVE: This research analyzed the clinical perspective of cognitive-behavioral therapy (CBT) discussed in the published scientific literature on the treatment of depression. METHOD: Through a bibliographic review on the Google Scholar Platform and the Scientific Electronic Library (SciELO) in the years 2010-2020. RESULTS AND DISCUSSIONS: The literature shows that resolving deadlocks more assertively, cognitive restructuring, and the focused psychotherapeutic process are understood as contributing to the effectiveness of treatment. It was noted that among the existing techniques in $\mathrm{CBT}$, some are more effective, namely: cognitive conceptualization, recording of dysfunctional thoughts, psychoeducation, cognitive restructuring, socratic questioning, social skills training, and homework. FINAL CONSIDERATIONS: This study has addressed the prevalence of depressive disorders, their developmental prospects, signs, and symptoms, as well as the management of treatment, and it can be inferred that CBT and the technical tools used by it corroborate the progress of psychotherapy and cognitive restructuring of depressive individuals, attenuating the symptoms, in partnership with the use of psychotropic drugs.
\end{abstract}

KEYWORDS: Cognitive-Behavioral Psychology. Depression. Clinical treatment. 


\section{Introducción}

En los últimos años ha aumentado considerablemente el número de estudios destinados a comprender mejor las enfermedades mentales. Dicho esto, a través de las investigaciones sobre los trastornos psicológicos, así como sus síntomas, se ha hecho posible entender cómo se desarrollan y, en consecuencia, mejorar las estrategias de tratamiento (Camargo et al., 2014). Sin embargo, este artículo tiene como objetivo analizar la perspectiva clínica de la terapia cognitivo-conductual (TCC) en la literatura científica publicada sobre el tratamiento de la depresión.

Según Camargo et al. (2014), la depresión se considera uno de los trastornos más frecuentes hoy en día, alcanzando, en promedio, entre el 15\% y el $20 \%$ de la población mundial, que afirma que millones de personas, en algún momento de su vida, han experimentado o experimentarán algún episodio depresivo. Además, según los autores, la depresión es una enfermedad que se presenta con características progresivas, es decir, ha causado, está causando y causará aún más daño a la salud de las personas afectadas por ella.

En esta perspectiva, Fédida (2014) destaca el predominio de los estudios psiquiátricos a lo largo de los años sobre los males causados a quienes padecen el trastorno depresivo, además de reconocerlo como una epidemia en la actualidad. Asimismo, la Organización Mundial de la Salud (Marcus et al., 2012) considera que la depresión es una de las enfermedades más frecuentes que afectan a la población mundial en la actualidad, causando problemas psíquicos y sociales. Por lo tanto, se supone que para 2030 la depresión estará a la cabeza de las psicopatologías, convirtiéndose en la principal causa de sufrimiento en las personas, caracterizando así un problema de salud pública.

A su debido tiempo, la Asociación Americana de Psiquiatría (APA, 2013) clasifica la depresión como un trastorno del estado de ánimo mental alterado, que trae como síntomas más comunes la sensación de vacío y tristeza asociada con la irritación y los pensamientos disfuncionales de discapacidad, de modo que la salud y la calidad de vida se ven comprometidas. Además, es una condición que se ve comprometida en la esfera social del individuo, causando dificultades en las relaciones interpersonales, así como provocando distorsiones en cuanto a su visión del mundo (Beck et al., 1997).

Basándose en la suposición de que las psicopatologías se originan en la forma en que el individuo percibe los eventos que le ocurren, Aaron Beck desarrolló el enfoque psicológico de la Terapia CognitivoComportamental (TCC). El autor lo formuló inicialmente centrándose en los pacientes depresivos, considerando que la depresión se caracteriza, en su lógica, como una visión negativa de uno mismo, del mundo y del futuro (Beck et al., 1997).

La TCC es un enfoque considerado directivo, centrado y estructurado, que se basa en la tríada compuesta por la cognición, o pensamiento, emoción y comportamiento. Así pues, esos elementos se influyen mutuamente, en los que la cognición se considera un factor esencial y conductor. De esta manera, los pensamientos disfuncionales provocan cambios en los sentimientos y las acciones que, en consecuencia, interfieren en el funcionamiento psíquico del individuo de manera inapropiada. Por lo tanto, esta perspectiva hace hincapié en la sustitución de esos pensamientos distorsionados por otros más adaptables y coherentes con la realidad, que proporcionen estrategias funcionales e influyan positivamente en todas las esferas de la vida del sujeto, como se abordará en el curso del presente artículo (Beck, 2013).

En vista de lo anterior, el presente estudio tiene por objeto evaluar, mediante un examen teórico, la forma en que la terapia cognitivo-comportamental ha sido eficaz para reducir las manifestaciones sintomáticas de la depresión. Así pues, el interés por el tema surgió del deseo del investigador de mejorar su comprensión del trastorno depresivo, teniendo en cuenta sus experiencias teóricas adquiridas durante su especialización en la terapia cognitiva comportamental. Por lo tanto, al considerar las consecuencias derivadas de este fenómeno psicológico y la complejidad de sus síntomas, surgieron las siguientes preguntas: ¿cuáles son las causas y síntomas comunes de la depresión? ¿Cuáles son las principales formas de tratamiento? ¿Cuál es el apoyo que presenta la terapia cognitivacomportamental? ¿Qué tan efectiva ha sido la TCC en el tratamiento de la depresión? 
Mientras tanto, la investigación se entiende como pertinente al contexto académico y social, buscando contribuir a la construcción de nuevos soportes teóricos, ampliando el conocimiento sobre la depresión y sus estrategias de tratamiento, dado el alarmante número de individuos afectados por dicha psicopatología en nuestra situación mundial. También, a través de este trabajo, pretendemos acercar a los investigadores, psicólogos y psicólogas, así como a la sociedad civil, al tema aquí tratado. Así pues, este estudio presenta dos bloques de discusión, en un primer momento una exploración conceptual de la historia de la psicopatología de la depresión, y luego una revisión bibliográfica en las plataformas de la Biblioteca Académica y Científica Electrónica de Google (SciELO), pertinente al tema, en el período mínimo de 2010 a 2020, con el fin de mapear el escenario de la discusión analítica de la depresión desde la perspectiva de la terapia cognitivo-comportamental.

\section{Depresión: comprender el trastorno a través de su historia}

En la era moderna, la psiquiatría entiende el trastorno depresivo como un trastorno psicológico, que causa problemas emocionales y afectivos y desajustes en las relaciones sociales y el entorno. De la misma manera, considera el mal funcionamiento bioquímico del cerebro como uno de los factores desencadenantes de este desorden de la mente (Solomon, 2002). Soares et al. (2014) señalan que la depresión es un trastorno de desequilibrio prolongado del estado de ánimo, cuya causa principal es la escasez de los neurotransmisores serotonina, noradrenalina y dopamina.

Para comprender la depresión es necesario examinar la sintomatología clínica del trastorno. Dependiendo de los síntomas de la depresión, incluyen la fragilidad humana, actividades lentas y desmotivadas, energía o disposición reducida, desinterés y concentración difícil, pérdida de la capacidad de planear, disfunción en el pensamiento con respecto a la realidad negativa y fuera de ella, y vulnerabilidad en la vida (Silva, 2015). Desde este ángulo, Biazus y Ramires (2012) destacan, además del factor desmotivador de larga duración que causa a los sujetos, características como el bajo estado de ánimo, la irritabilidad con tendencia a estallar de ira y los frecuentes problemas psicosociales.

Aún sobre los síntomas manifestados, tenemos los pensamientos constantes de inutilidad y culpabilidad, baja autoestima, falta de interés en las actividades psicomotoras, incluyendo problemas de fatiga, insomnio, deseo de muerte, baja concentración, estado de ánimo deprimido constante y persistente que dura por lo menos dos semanas, como factores para la clínica depresiva (Asociación Americana de Psicologia, 2013). Así pues, la depresión se caracteriza por ser un trastorno que causa sufrimiento psíquico, fisiológico y social, generando así diversas pérdidas en la vida de los sujetos afectados.

En vista de los daños causados por el trastorno depresivo, también se considera que es de naturaleza existencial. Las creencias negativas son factores que potencian la depresión, por lo tanto, oportunizan una crisis vital y patológica en los individuos (Pacciolla, 2014). Corroborando con los autores mencionados, Santos y Faro (2015) subrayan que la crisis existencial corresponde a una baja autoestima, que es uno de los muchos indicadores del trastorno. Por lo tanto, las disfunciones presentadas, siendo éstas externalizadas a través del habla o incluso de la expresión corporal, son reflejos de un vacío interno.

Aunque los estudios apuntan a factores biológicos, psicológicos y sociales, no es posible determinar con precisión cuál es la principal causa de la depresión. Sin embargo, como señala Kolvalski (2015), la depresión no se caracteriza por ser un síntoma primario, sino más bien secundario, destacando también que las causas traumáticas relacionadas con factores emocionales aparecen como desencadenantes del trastorno. Para Sousa (2014), la depresión se presenta como una comorbilidad en los trastornos de ansiedad, es decir, primero el sujeto sufre algún trauma para, en consecuencia, desarrollar conductas de escape y evasión $y$, finalmente, entrar en un cuadro depresivo.

Desde el punto de vista fisiológico, es necesario comprender los posibles desencadenantes de la depresión, porque, según Chaki y Fukumoto (2015), los síntomas depresivos están directamente influenciados por los transmisores de monoamina como la serotonina, la noradrenalina y la dopamina, 
que actúan sobre el sistema neuroendocrino, provocando una situación clínica. A través de la disminución de los neurotransmisores serotonina, noradrenalina y dopamina nuestra capacidad funcional fisiológica pasa a un estado crónico, afectando el estado de ánimo y causando síntomas depresivos (Rueda, 2014).

\section{La terapia cognitiva-comportamental en el tratamiento de la depresión}

En cuanto al tratamiento psicoterapéutico de la depresión con énfasis en la Terapia CognitivoComportamental, es fundamental contar con una alianza terapéutica consolidada que favorezca el vínculo entre ambos -terapeuta y paciente- para asegurar la eficacia del proceso (Beck, 2013). Desde esta perspectiva, el terapeuta utilizará recursos y técnicas específicas para el seguimiento, considerando la conceptualización cognitiva realizada inicialmente, ya que sólo a partir de ésta el profesional tendrá conocimiento y dominio de la demanda presentada por el paciente (Cizil \& Beluco, 2019).

No obstante, teniendo en cuenta la complejidad de los trastornos depresivos, así como los cambios en las funciones psíquicas que se derivan de ellos, Dalgalarrondo (2008) señala que las principales funciones que se ven afectadas se refieren a la cognición, los estados de ánimo -por ejemplo, la melancolía y la tristeza- y las actividades volitivas. Dicho esto, el tratamiento de psicoterapia cognitivocomportamental se centrará en la cognición para que, en consecuencia, las demás funciones produzcan mejoras clínicas significativas, considerando así la tríada recomendada por el enfoque (Beck, 2013; Greenberger \& Padesky, 2017).

Además, la activación del comportamiento se utiliza comúnmente en pacientes con un trastorno depresivo grave, ya que, debido a las distorsiones cognitivas que presentan, su pragmatismo sufrirá daños, no sólo necesitando reestructurar la cognición. Así, a partir de la identificación del Pensamiento Automático disfuncional (PCA), el psicoterapeuta cognitivocomportamental actuará buscando comprender las actividades que el paciente entiende en la condición de agradable y desagradable, qué acciones solía realizar y ya no se esfuerza por hacer, en detrimento de la psicopatología. A partir de entonces, se iniciará una planificación de actividades, de manera práctica y efectiva, que apunte al proceso de activación comportamental, en el que el paciente desarrollará conductas rutinarias, de acuerdo con la orientación terapéutica y el acuerdo entre ambos (Beck, 2013).

Para ello se utilizan habitualmente recursos terapéuticos como la psicoeducación, los deberes, los registros de pensamientos disfuncionales (DPR), la reestructuración cognitiva, el cuestionamiento socrático, la capacitación en habilidades sociales, entre otros. Además, se evalúa la asociación entre la psicoterapia y la farmacoterapia a fin de satisfacer las demandas presentadas y alcanzar los objetivos fijados para garantizar mejoras sustanciales en la calidad de vida y la salud psíquica del paciente (Beck, 2013).

Por lo tanto, Beck y Dozois (2010) sugieren que, en los casos de síntomas psicóticos, el uso de técnicas de TCC en combinación con la farmacoterapia es eficaz para reducir los síntomas depresivos, la ansiedad, los pensamientos suicidas, los pensamientos automáticos negativos, entre otros, proporcionando un mejor funcionamiento en la vida de los sujetos. Por lo tanto, Kaplan y Sadock (2007), al considerar los diversos tipos de trastornos depresivos, basados en la depresión mayor, presentan entre los síntomas más característicos la aparición de alucinaciones y delirios, por lo que es indispensable contar con una combinación de medicamentos antidepresivos y antipsicóticos en casos específicos. Así pues, aunque la TCC es un enfoque indicado para el tratamiento de la depresión, es necesario combinarla con drogas psicoactivas.

\section{Metodología de revisión de literatura}

El camino metodológico que ha guiado esta investigación se basó en la revisión narrativa de la literatura. La selección y narración de los contenidos se configuran como cualitativos, amplios y con sus variables (Marconi \& Lakatos, 2017). En vista de ello, esta vía metodológica nos permite discutir, dialogar con marcos teóricos y contextuales sobre cuestiones específicas, para poder ser descrita como metodología adecuada. Por lo tanto, se trata de una revisión narrativa, cuyo propósito es buscar una breve descripción de cómo la Terapia Cognitivocomportamental aplica sus principales técnicas en el 
tratamiento de la depresión y así reflexionar sobre su eficacia.

La investigación cualitativa, según Minayo (2013), tiene en cuenta la investigación en las experiencias y vivencias de los sujetos, considerando el lenguaje influenciado por la cultura como estructuras fundamentales. La historia de la vida de los sujetos, sus relaciones, creencias, significados, valores asignados, deseos y acciones, sitúan la investigación cualitativa al nivel de la realidad de las personas.

Teniendo en cuenta que las intervenciones que la TCC realiza en la atención clínica se basan en herramientas que pretenden mejorar el funcionamiento psíquico $y$, en consecuencia, la calidad de vida del individuo, buscamos aquí el esclarecimiento de algunas investigaciones sobre el trastorno depresivo y la visión que la TCC tiene de él. La centralidad de este estudio, como se mencionó anteriormente como objetivo, es tematizar por medio de la literatura científica este escenario de interlocuciones entre la TCC y la depresión. Así, además del uso de la bibliografía clásica, esta revisión se realizó mediante la búsqueda de artículos en revistas electrónicas como Google Scholar y la Biblioteca Científica Electrónica (SciELO), relevantes para el tema, en el período mínimo de 2010 a 2020.

Al no tratarse de una revisión bibliográfica sistemática, La elección de las bases de datos se justificó por el libre acceso a los estudios con revistas indexadas en estos portales, y la cobertura nacional con ejes de publicaciones de diversas áreas del conocimiento, además de ser las bases de investigación más importantes de latinoamérica. La elección del marco temporal se basó en la intención de producir una investigación bibliográfica narrativa de los últimos diez años contados a partir de la fecha de redacción de este estudio. Los descriptores utilizados para la búsqueda fueron "cognitive-behavioral therapy" AND "depression". La recopilación de los estudios publicados en las bases de datos se realizó mediante un muestreo aleatorio no probabilístico, es decir, seleccionado a partir de la vinculación con el tema, y cesado a partir del criterio de saturación dentro del corte temporal, y bajo correspondencias del objetivo rector de este estudio.

Los criterios de inclusión fueron los artículos publicados en los últimos diez años, considerando el año en curso, en portugués y en lenguas extranjeras, que abarcaran el tema estudiado. Así, para los criterios de exclusión, tenemos los artículos que no estaban dentro del tiempo preestablecido de publicación, las publicaciones incompletas o no disponibles para el acceso público, los estudios que no tenían como centralidad el uso clínico de la TCC, y por último también, los que no contemplaban el objetivo de este estudio. Parte de la selección de los estudios y la recuperación de las bases de datos fue la participación de 4 autores de este estudio, cuya experiencia con el tema y el método están en la formación de pregrado y postgrado en Psicología, así como la publicación en los métodos de revisión narrativa de la literatura y la revisión sistemática de la literatura. En la siguiente sección, se analiza una síntesis de los estudios recuperados en nuestras búsquedas bibliográficas.

\section{Resultados y discusiones}

La terapia cognitiva-comportamental se ha formulado para abordar la cognición distorsionada, es decir, los pensamientos y creencias que están alejados de la realidad. Además, cabe señalar que su precursor, Aaron Beck, lo propuso apuntando, en un primer momento, al tratamiento de los pacientes depresivos, al darse cuenta de la necesidad de crear una forma corta y directa de manejar ese trastorno (Cizil \& Beluco, 2019). En general, la TCC es un enfoque que se presenta con una gama de técnicas psicoterapéuticas que tienen por objeto trabajar en el manejo de diversos trastornos psicológicos. En cuanto a la depresión, Cuijpers (2016) señala que tales herramientas psicoterapéuticas surgen en primera línea como tratamiento clínico, es decir, con gran eficacia.

Este enfoque psicoterapéutico se ha ido introduciendo gradualmente en la práctica clínica. Su gestión hace hincapié en la búsqueda de pruebas frente a la cognición y el análisis de la correlación existente entre la cognición, las emociones y las conductas, con el fin de ayudar a los sujetos a reducir el sufrimiento psíquico. Por lo tanto, tiene como objetivo identificar los pensamientos más comunes, así como sus posibles distorsiones y ayudar a modificarlos (Wright et al., 2008; Cizil \& Beluco, 2019). 
La Organización Mundial de la Salud (Organización Mundial de la Salud, 2012) destaca que se estima que 350 millones de personas en el mundo se verán afectadas por la depresión, con un rango sintomático de por vida de entre el 3 y el 17\%. En este período, es común que haya pensamientos y comportamientos estandarizados en las personas afectadas por el trastorno. De esta manera, es notoria la percepción distorsionada de uno mismo, de los demás y del mundo, en la que el sujeto utiliza comúnmente afirmaciones como: "No sé hacer nada", "no le gusto a nadie", "soy aburrido", "nunca me mejorarán las cosas", "no soy una persona interesante", entre otras. Los errores cognitivos son elementos que están demasiado presentes en las personas depresivas (Carneiro \& Dobson, 2017).

En resumen, las distorsiones cognitivas están presentes en la cognición de los sujetos afectados por la depresión. Según Carneiro y Dobson (2017), estas distorsiones provienen de las creencias centrales y subyacentes que se configuran desde la infancia y se extienden a lo largo de la vida. En sus estudios sobre la depresión, Beck (2013) destacó la necesidad de que el terapeuta examine más de cerca algunos errores cognitivos, entre ellos la llamada inferencia arbitraria, que coopera para determinar algo que existe sin pruebas. La supergeneralización es una distorsión que favorece la generalización del sujeto a una visión negativa de los acontecimientos que experimenta, mientras que en la personalización el individuo se autoconsidera una persona negativa, debido a los pensamientos automáticos que pasan repetidamente por su cognición (Beck, 2013).

En vista de estos atenuantes, el terapeuta cognitivocomportamental utilizará las técnicas de TCC para realizar el manejo clínico. Para Beck y otros (1997), la reestructuración cognitiva, la psicoeducación y la capacitación en aptitudes sociales deben ser las principales técnicas de trabajo en las personas con depresión. La reestructuración cognitiva permite trabajar gradualmente en los patrones de cognición, emociones y comportamiento de los sujetos. La educación psicosocial está diseñada para enseñar sobre el modelo cognitivo, el trastorno y sus síntomas, mientras que el entrenamiento de habilidades sociales ayudará al paciente a desarrollar un repertorio comportamental funcional para actuar y comportarse de manera más asertiva (Cizil \& Beluco, 2019).
En este sentido, entre las técnicas consideradas efectivas en el manejo clínico de la depresión se encuentra también la conceptualización cognitiva, una herramienta de apoyo para que el terapeuta lo guíe en el contexto del paciente $y$, de esta manera, programe sus intervenciones con mayor seguridad. Por lo tanto, la conceptualización permite una mejor hipótesis diagnóstica al cartografiar la historia de la vida del sujeto (Cizil \& Beluco, 2019).

Dicho esto, al considerar la historia de la vida de las personas para una mejor comprensión del trastorno es necesario prestar atención a su carácter multifacético. Por lo tanto, una sola forma de tratamiento no comprende la misma eficacia con todas las personas. Además, en varios casos, se necesita una combinación farmacológica específica para la demanda de cada sujeto (Dobson, 2016).

De esta manera, se percibe el reconocimiento de la TCC como eficaz en la práctica psicoterapéutica del tratamiento del trastorno depresivo. Como señalan Matos y Oliveira (2013), este enfoque permite resolver los problemas de una manera más eficaz que otras perspectivas teóricas. Sin embargo, incluso con eficacia comprobada, el enfoque por sí solo no garantiza una "cura", o un tratamiento $100 \%$ eficaz, y es esencial comprender que el trastorno depresivo, en la mayoría de los casos, requiere una combinación de seguimiento psicológico asociado a la terapia con medicamentos (Matos \& Oliveira, 2013).

Por lo tanto, cuando se piensa en tratamientos para la depresión que están disociados, es decir, que utilizan sólo psicoterapia o sólo fármacos, hay variaciones en los resultados. Un estudio en el que se reclutaron 166 pacientes, de entre 19 y 74 años de edad, diagnosticados con el trastorno de depresión grave (MDD), mostró que los pacientes que fueron tratados sólo con TCC tuvieron mayores resultados positivos que los que fueron seguidos sólo con el uso convencional de drogas (Lopez \& Basco, 2015).

Un segundo estudio realizado por Lam y otros (2013) durante un período de 12 semanas con pacientes diagnosticados con MDT, de 19 a 65 años de edad, tenía por objeto comparar los resultados obtenidos con un grupo de pacientes que recibieron sólo la intervención farmacológica con antidepresivos y otro grupo que recibió la misma medicación asociada a la TCC. 
Los resultados mostraron una mayor eficacia en el segundo grupo. Así pues, se concluye que la práctica de la TCC con intervenciones de drogas es significativamente competente en la remisión de los síntomas depresivos (Lam et al., 2013).

Por último, considerando que la TCC busca a través de la técnica de conceptualización cognitiva comprender cómo funciona el sujeto en el mundo y cuál es el contexto de su historia de vida, se destaca que, a través de la técnica de reestructuración cognitiva, la psicoeducación y otras estrategias desde esta perspectiva teórica se ofrece un apoyo que ayuda a los individuos a tener una mejor comprensión del modelo cognitivo y de los problemas que están experimentando. De esta manera, se basa en estudios que demuestran su eficacia en el tratamiento de la depresión que la investigación contempla sus objetivos frente a los resultados obtenidos, estando indicado para personas afectadas por el trastorno depresivo en sus más diversos síntomas, ya que el psicoterapeuta cognitivo-comportamental servirá de apoyo al público en cuestión.

\section{Consideraciones finales}

El presente estudio tuvo por objeto presentar, sobre la base de aportaciones teóricas, la eficacia de la terapia cognitivo-comportamental (TCC) en el tratamiento clínico de las personas afectadas por la depresión. Habida cuenta de que muchos consideran que ese enfoque es una práctica psicológica mecanicista, se pudo concluir que la TCC es eficaz en el tratamiento de los trastornos depresivos y en la remisión de sus síntomas. Los estudios de revisión de la literatura densifican la lectura sobre el desarrollo científico de un tema dado, en este estudio se observa que la TCC se ha colocado como una estrategia importante para el afrontamiento de la depresión, sin embargo, es necesario ampliar el análisis en otras bases de datos e inclusión de datos del análisis de estudios originales.

Por lo tanto, se puede decir que la TCC es una línea teórica estructurada que tiene como objetivo hacer del cliente su propio terapeuta. Así pues, como se señala en este trabajo, las técnicas utilizadas ayudan al terapeuta en la conducción del proceso psicoterapéutico $y$, por consiguiente, en la reestructuración cognitiva de los pacientes, especialmente los afectados por la depresión en sus más variados atenuantes y síntomas. En este sentido, es pertinente señalar su mayor eficacia cuando se asocia con la intervención de drogas en estos casos.

En cuanto a la realización de la investigación, es pertinente señalar que las limitaciones de tiempo y cantidad de hallazgos teóricos para el desarrollo de la revisión de la literatura y la construcción de un marco teórico estuvieron presentes en su desarrollo, requiriendo un mayor compromiso del investigador para lograr sus objetivos. Por último, se muestra la pertinencia de este estudio tanto en la formación académica del investigador como en el ámbito científico y social, con miras a la publicación de los resultados obtenidos $y$, en consecuencia, a la aclaración a la sociedad de la existencia de tratamientos eficaces para la remisión del sufrimiento y los daños causados por la depresión. Sin embargo, se reconoce la amplia complejidad de este trastorno y se sugiere el desarrollo de nuevos estudios que proporcionen una mayor comprensión de la depresión y el uso de la terapia cognitivacomportamental en su tratamiento.

\section{Contribuciones de los autores}

Teixeira, C. R. participó en la concepción, diseño, búsqueday análisis cualitativo de los datos de la investigación, interpretación de los resultados, redacción del artículo científico. Alves, T. P. participó en la concepción, diseño, interpretación de resultados, redacción y envío del trabajo científico. Teixeira, M. W. S., Lavor Filho, T. L., Silva, L. M., Gomes Filho, A. S. participaron en la recopilación de datos de investigación, la interpretación de datos, la redacción del artículo científico.

\section{Conflitos de intereses}

No se ha declarado ningún conflicto financiero, legal o político con terceros (gobierno, empresas privadas y fundaciones, etc.) para ningún aspecto del trabajo presentado (incluyendo, pero sin limitarse a las subvenciones y la financiación, la participación en el consejo asesor, el diseño del estudio, la preparación del manuscrito, el análisis estadístico, etc.). 


\section{Referências}

Abreu, N., \& Oliveira, I. R. (2008). Terapia cognitiva no tratamento da depressão [La terapia cognitiva en el tratamiento de la depresión]. In A. V. Cordioli (Ed.), Psicoterapias: abordagens atuais (pp. 382-898). Artmed.

American Psychiatric Association - APA. (2013). DSM-5: manual diagnóstico e estatístico de transtornos mentais $\left(5^{\mathrm{a}}\right.$. ed.) [Manual Diagnóstico y Estadístico de Trastornos Mentales]. Artmed.

Beck, A. T., Rush, A. J., Shaw, B. F., \& Emery, G. (1997). Terapia cognitiva da depressão [Terapia cognitiva de la depresión]. Artmed.

Beck, A. T., \& Dozois, D. J. (2010). Cognitive therapy: current status and future directions [Terapia cognitiva: estado actual y direcciones futuras]. Annual review of medicine, 62, 397409. https://doi.org/10.1146/annurev-med-052209-100032

Beck, J. S., \& Beck, A. T. (2011). Cognitive therapy: Basics and beyond [La terapia cognitiva: Lo básico y más allá]. Guilford press.

Beck, J. (2013). Terapia Cognitivo-Comportamental: Teoria e Prática [Terapia cognitivo-conductual: teoría y práctica] (2a ed.). Artmed.

Berlinck, M. T., \& Fédida, P. (2014). A clínica da depressão: questões atuais [La clínica de la depresión: cuestiones actuales]. Revista Latinoamericana de psicopatologia fundamental, 3(2), 9-25. https://doi.org/10.1590/141547142000002002

Biazus, C. B., \& Ramires, V. R. R. (2012). Depression in adolescence: a problematic of bonds [La depresión en la adolescencia: una problemática de vínculos]. Psychology in Study, 17(1), 83-91. https://www.scielo.br/j/pe/a/ RSkXKnYD4frXYKQrmzGdGMn/abstract/?lang=pt

Caballo, V. E. (2003). Manual de avaliação e treinamento das habilidades sociais [Manual de evaluación y formación en habilidades sociales]. Santos.

Caballo, V. E. (2002). Manual para o tratamento cognitivo comportamental: transtornos de ansiedade, sexuais, afetivos e psicóticos [Manual de tratamiento cognitivo-conductual: trastornos de ansiedad, sexuales, afectivos y psicóticos]. Santos.

Camargo, R. M., Sousa, C. O., \& Oliveira, M. L. C. (2014). Prevalência de casos de depressão em acadêmicos de enfermagem em uma instituição de ensino de Brasília [Prevalencia de casos de depresión en estudiantes de enfermería de una institución educativa de Brasilia]. Revista Mineira de Enfermagem, 18(2), 392-403. http://www. reme.org.br/artigo/detalhes/935
Carneiro, A. M., \& Dobson, K. S. (2016). Tratamento cognitivocomportamental para depressão maior: uma revisão narrativa [Tratamiento cognitivo-conductual para la depresión mayor: una revisión narrativa]. Revista Brasileira de Terapias Cognitivas, 12(1), 42-49. https://dx.doi. org/10.5935/1808-5687.20160007

Chaki, S., \& Fukumoto, K. (2015). Potential of glutamate-based drug discovery for next generation antidepressants [Potencial del descubrimiento de fármacos basados en el glutamato para la próxima generación de antidepresivos]. Pharmaceuticals, 8(3), 590-606. https://dx.doi. org/10.3390\%2Fph8030590

Cizil, M. J., \& Beluco, A. C. R. (2019). As contribuições da terapia cognitivo comportamental no tratamento da depressão [Las aportaciones de la terapia cognitivo-conductual en el tratamiento de la depresión]. Uningá Journal, 56(S1), 33-42. http://revista.uninga.br/index.php/uninga/article/view/88

Cuijpers, P. (2016). Are all psychotherapies equally effective in the treatment of adult depression? The lack of statistical power of comparative outcome studies [iSon todas las psicoterapias igual de eficaces en el tratamiento de la depresión en adultos? La falta de potencia estadística de los estudios comparativos de resultados]. Evidence-Based Mental Health, 19(2), 39-42. https://doi.org/10.1136/eb2016-102341

Dalgalarrondo, P. (2018). Psicopatologia e Semiologia dos Transtornos Mentais [Psicopatología y semiología de los trastornos mentales]. Artmed.

Del Prette, Z. A., \& Del Prette, A. (2017). Competência social e habilidades sociais: Manual teórico-prático [Competencia social y habilidades sociales: manual teórico y práctico] Vozes.

Dobson, K. S. (2016). New frontiers in cognitive-behavioral therapy for depression [Nuevas fronteras en la terapia cognitivoconductual para la depression]. International Journal of Cognitive Therapy, 9(2), 107-123. https://psycnet.apa.org/ doi/10.1521/ijct.2016.9.2.107

Fernandes, C. S., Falcone, E. M. O., \& Sardinha, A. (2012). Deficiências em habilidades sociais na depressão: estudo comparativo [Déficit de habilidades sociales en la depresión: un estudio comparativo]. Revista Psicologia-Teoria e Prática, 14(1), 183-183. http://pepsic.bvsalud.org/scielo.php?script=sci_ arttext\&pid=S1516-36872012000100014.

Gottschalk, C. M. C. (2010). O papel do método no ensino: da maiêutica socrática à terapia wittgensteiniana [El papel del método en la enseñanza: de la mayéutica socrática a la terapia wittgensteiniana]. ETD: Digital Thematic Education, 12(1), 64-81. https://dialnet.unirioja.es/descarga/ articulo/4856510.pdf 
Greenberger, D., \& Padesky, C. A. (2017). A mente vencendo o humor: mude como você se sente, mudando o que você pensa [Mente que supera el estado de ánimo: cambia lo que sientes cambiando lo que piensas]. Artmed.

Hamermesh, D. S., \& Soss, N. M. (1974). An economic theory of suicide. Journal of Political Economy, 82(1), 83-98. https:// www.journals.uchicago.edu/doi/abs/10.1086/260171

Sadock, B. J., Sadock, V. A., \& Ruiz, P. (2007). Compêndio de Psiquiatria: Ciência do Comportamento e Psiquiatria Clínica [Compendio de psiquiatría: ciencias del comportamiento y psiquiatría clínica]. Artmed.

Knapp, P. (2004). Terapia Cognitivo Comportamental na Prática Psiquiátrica [La terapia cognitivo-conductual en la práctica psiquiátrica]. Artmed.

Knapp, P., \& Beck, A. T. (2008). Fundamentos, modelos conceituais, aplicações e pesquisa da terapia cognitiva [Fundamentos, modelos conceptuales, aplicaciones e investigación de la terapia cognitiva]. Revista brasileira de psiquiatria, 30 Suppl 2, s54-s64. https://doi.org/10.1590/ s1516-44462008000600002

Kuyken, W., Padesky, C. A., \& Dudley, R. (2010). Conceituação de casos colaborativa: $O$ trabalho em equipe com pacientes em terapia cognitivo-comportamental [Conceptualización colaborativa del caso: Trabajo en equipo con pacientes en terapia cognitivo-conductual]. Artmed.

Lam, R. W., Parikh, S. V., Ramasubbu, R., Michalak, E. E., Tam, E. M., Axler, A., Yatham, L. N., Kennedy, S. H., \& Manjunath, C. V. (2012). Effects of combined pharmacotherapy and psychotherapy for improving work functioning in major depressive disorder [Efectos de la farmacoterapia y la psicoterapia combinadas para mejorar el funcionamiento laboral en el trastorno depresivo mayor]. The British Journal of Psychiatry, 203(5), 358-365. https://doi. org/10.1192/bjp.bp.112.125237

Lopez, M. A., \& Basco, M. A. (2015). Effectiveness of cognitive behavioral therapy in public mental health: Comparison to treatment as usual for treatment-resistant depression [Eficacia de la terapia cognitivo-conductual en la salud mental pública: Comparación con el tratamiento habitual para la depresión resistente al tratamiento]. Administration and policy in mental health and mental health services research, 42(1), 87-98. https://dx.doi. org/10.1007\%2Fs10488-014-0546-4

Marback, R. F., \& Pelisoli, C. (2014). Terapia cognitivocomportamental no manejo da desesperança e pensamentos suicidas [Terapia cognitivo-conductual en el manejo de la desesperanza y las ideas suicidas]. Revista Brasileira de Terapias Cognitivas, 10(2), 122-129. http:// dx.doi.org/10.5935/1808-5687.20140018

Marconi, M. D. A., \& Lakatos, E. M. (2017). Metodologia científica [Metodología científica] (Vol. 4). Atlas.
Marcus, M., Yasamy, M. T., Ommeren, M. V., \& Chisholm, D. (2012). Depression: A global public health concern [La depresión: Un problema de salud pública mundial]. Organización Mundial de la Salud. https://www.who.int/mental health/ management/depression/who_paper_depression wfmh_2012.pdf

Matos, A. C. S., \& Oliveira, I. R. (2013). Terapia cognitivocomportamental da depressão: relato de caso [Terapia cognitivo-conductual de la depresión: informe de un caso]. Revista de Ciências Médicas e Biológicas, 12(4), 512-519. http://dx.doi.org/10.9771/cmbio.v12i4.9203

Mercês, E. L., Moura, L. F., \& Oliveira, I. J. (2018). Terapia cognitivocomportamental aplicada à depressão: uma breve revisão bibliográfica [Terapia cognitivo-conductual aplicada a la depresión: una breve revisión de la literatura]. Amazônia Science \& Health Journal, 6(1), 2-11. http://www.ojs.unirg. edu.br/index.php/2/article/view/1321/pdf 2

Minayo, M. C. S. (2013). O desafio do conhecimento - pesquisa qualitativa em saude [El reto del conocimiento: la investigación sanitaria cualitativa] (13th ed.). HUCITEC. https://pesquisa.bvsalud.org/portal/resource/pt/crt-1255

Pacciolla, A. (2014). DSM-5 e temi esistenziali [DSM-5 y cuestiones existenciales]. Laurus Robuffo.

Rueda, F. J. M., Alves, S. M. M., \& Baptista, M. N. (2014). Evidência de validade preliminar da escala de depressão (EDEP): um estudo com alunos de enfermagem [Pruebas de validez preliminar de la escala de depresión (EDEP): un estudio con estudiantes de enfermería]. Psicologia Argumento, 32(79). https://periodicos.pucpr.br/index.php/ psicologiaargumento/article/download/20435/19695

Santos, L. C. S., \& Faro, A. (2015). Relações entre Autoestima e Sentido de vida: Estudo com amostragem domiciliar em Aracaju (SE) [Relaciones entre autoestima y sentido de la vida: Estudio con muestreo de hogares en Aracaju (SE)]. Clínica \& Cultura, 4(2), 54-69. https://ri.ufs.br/handle/ riufs $/ 1915$ ?locale $=$ en

Santos, C. E. M., \& Medeiros, F. A. (2017). A relevância da técnica de questionamento socrático na prática CognitivoComportamental [La relevancia de la técnica del cuestionamiento socrático en la práctica cognitivoconductual]. Archives of health investigation, 6(5), 204-208. https://doi.org/10.21270/archi.v6i5.1940

Silva, D. S. D., Tavares, N. V. S., Alexandre, A. R. G., Freitas, D. A. Brêda, M. Z., Albuquerque, M. C. S., \& Melo Neto, V. L. (2015). Depressão e risco de suicídio entre profissionais de Enfermagem: revisão integrativa [Depresión y riesgo de suicidio entre los profesionales de enfermería: revisión integradora]. Revista da Escola de Enfermagem da USP, 49(6), 1023-1031. https://doi.org/10.1590/S0080$\underline{623420150000600020}$ 
Soares, V. C., Nascimento, B. R., Viana, T. R., Lopes, N. P., \& Franco, A. J. (2016). Análise da prescrição de paroxetina em uma drogaria do município de ponte nova, minas gerais [Análisis de la prescripción de paroxetina en una farmacia de ponte nova, minas gerais]. ANAIS SIMPAC, 6(1). https://academico.univicosa.com.br/revista/index.php/ RevistaSimpac/article/view/473

Solomon, A. (2002). O demônio do meio-dia: uma anatomia da depressão [El demonio del mediodía: una anatomía de la depresión]. Objetiva.
Sousa, M. C. C. (2014). Comorbidade e relação temporal entre ansiedade e depressão em idosos institucionalizados [Comorbilidad y relación temporal entre ansiedad y depresión en ancianos institucionalizados] [Tesis de maestría, Instituto superior Miguel Torga, Escola superior de altos estudos]. DSpace Repository. http://repositorio. ismt.pt/xmlui/handle/123456789/451

Wright, J. H., Brown, G. K., Thase, M. E., \& Basco, M. R. (2008). Aprendendo a Terapia Cognitivo-Comportamental: Um Guia Ilustrado [Aprendizaje de la terapia cognitivo-conductual: una guía ilustrada]. Artmed. 